\title{
Design and analysis of a bio-inspired module-based robotic arm
}

\author{
Zirong Luo ${ }^{1}$, Jianzhong Shang ${ }^{1}$, Guowu $\mathrm{Wei}^{2}$, and Lei Ren ${ }^{3}$ \\ ${ }^{1}$ School of Mechatronics Engineering and Automation, National University of Defence Technology, \\ 410073 Changsha, China \\ ${ }^{2}$ School of Computing, Science and Engineering, University of Salford, Salford, M5 4WT, UK \\ ${ }^{3}$ School of Mechanical, Aerospace and Civil Engineering, University of Manchester, \\ Manchester, M13 9PL, UK
}

Correspondence to: Zirong Luo (luozirong@nudt.edu.cn), Guowu Wei (g.wei@salford.ac.uk) and Lei Ren (lei.ren@manchester.ac.uk)

Received: 30 March 2016 - Revised: 4 July 2016 - Accepted: 11 July 2016 - Published: 12 August 2016

\begin{abstract}
This paper presents a novel bio-inspired modular robotic arm that is purely evolved and developed from a mechanical stem cell. Inspired by stem cell whilst different from the other robot "cell" or "molecule", a fundamental mechanical stem cell is proposed leading to the development of mechanical cells, bones and a Sarrus-linkage-based muscle. Using the proposed bones and muscles, a bio-inspired modular-based five-degreesof-freedom robotic arm is developed. Then, kinematics of the robotic arm is investigated which is associated with an optimization-method-based numerical iterative algorithm leading to the inverse kinematic solutions through solving the non-linear transcendental equations. Subsequently, numerical example of the proposed robotic arm is provided with simulations illustrating the workspace and inverse kinematics of the arm. Further, a prototype of the robotic arm is developed which is integrated with low-level control systems, and initial motion and manipulation tests are implemented. The results indicate that this novel robotic arm functions appropriately and has the virtues of lower cost, larger workspace, and a simpler structure with more compact size.
\end{abstract}

\section{Introduction}

In the field of robotics, researchers and engineers have a dream of developing robotic arms with agile characteristics like the human arm to perform dynamic tasks under complicated and unconstructed environment. However, more than sixty years passed since the first robotic manipulator being patented by George G. Devol in 1954 (see Siciliano and Khatib, 2008), it is still too far to say that this dream has come true. Nevertheless, new ideas and concepts have been putting forward to explore the ways of developing more efficient, dexterous and feasible robotic arms.

In order to achieve desired flexibility, manipulability and reconfigurability, one of the efforts is devoted to the development of novel, high performance module-based robotic arms. These include, to mention but a few, the one degree of freedom T-type and I-type modules proposed by Guan et al. (2009); the three degrees of freedom tendon-integrated spherical modules designed by Guckert and Naish (2009); the three degree of freedom active module suitable for space hyper-redundant robot presented by Shammas et al. (2006) and the bi-articular muscles developed by Yoshida et al. (2009); and through the construction of link modules and joints modules, Acaccia et al. (2008) developed a modular robotic system for generic industrial applications. However, most of these modules proposed are serial and rigid, they can only change the position and attitude of the endeffector within the allowable range of the linkage member. The degrees of flexibility of the structures of such serial rigid robotic arms are limited with simple functions such that they cannot adapt to the change of working environment and various customer demands. To overcome the shortcoming of serial robotic arms, effort has also been made to develop robotic arms based on parallel mechanism. Liu et al. (2006) proposed a novel five degree of freedom reconfigurable hy- 
brid robotic arm composed of a 2-DOF parallel mechanism and a 3-DOF serial kinematic chain. Jin and Gao (2002) studied a new 6-SPS parallel manipulator with an orthogonal configuration. Wurst and Peting (2002) presented PKM concept for designing modular-based reconfigurable machine tools, and using slide module and swing module, Xi et al. (2006) proposed reconfigurable parallel robot consisting of two base tripods.

In addition, bio-inspired robotic arms have also been designed and developed. One of the earliest bio-inspired robot is a new robotic system coined DRRS (Dynamically Reconfigurable Robotic System) proposed by Fukufda and Nakagawa (1988). This robotic system consists of a number of intelligent cells each of which has a fundamental mechanical function. Each cell can detach or connect itself to the other cells autonomously depending on a specified task, the robot can then be of a manipulator or a mobile robot, the system also has the function of self-repair. In 1990, Fukuda and Kawauchi (1990) further proposed a new kind of robotic system called CEBOT (The Cellular Robotic System), which is a distributed robotic system consisting of reconfigurable autonomous units called "cell"s, the robot can reconfigure itself to an optimal structure depending on the specified purpose and environment. Further, Kotay and Rus (1999) proposed a robotic module called "Molecule" to build selfreconfiguring robots, the Molecules support multiple modalities of locomotion and manipulation. In the same period, Rus and Vona (1999) discussed a robotic system composed of crystalline modules that can aggregate together to form distributed robot systems. The crystalline modules can also move relative to each other by expanding and contracting. This actuation mechanism permits automated shape metamorphosis and provides a crystalline robot system the capability of self-reconfiguration. More recently, Yim et al. (2000) presented a modular reconfigurable robot called PolyBot. PolyBot is a modular reconfigurable robot system composed of two types of modules serving as segments and nodes. A segment module has one degree of freedom and two connection ports, and a node module is rigid with no mobility and has six connection ports. It was reported that two PolyBot systems have been built, and a robot with up to 32 modules being bolted together have been tested.

In this paper, we propose a stem-cell-inspired mechanical robotic arm. Different from the robotic arms discussed above that each of their mechanical cells or molecules is not separated but a completed robotic module being integrated with control and software system, the mechanical stem cell proposed in this paper, inspired by the character of stem cell, at a more basic and fundamental level, is a type of pure mechanical unit. This unit can be used to build different robotic modules in according to the practical applications.

Using the unit, a bio-inspired mechanical muscle based on Sarrus linkage is proposed, leading to the development of a 5DOF bio-inspired robotic arm. This novel design helps augment workspace and overcome some shortcomings of con- ventional serial robotic arms. According to the available literature, although Sarrus linkage (Lee, 1996) has many applications in the field of robotics, including the crawling robot by Ranjana et al. (2006), the road vehicle by Gavin and Luis (2010) and the micro six-legged segmented robot by Katie and Robert (2011), there is no report on robotic arm that is constructed with Sarrus-linkage-based structures.

In this paper, a robotic arm evolved from a fundamental mechanical stem cell is for the first proposed, and its associated kinematics, simulation, prototype and initial test results are presented.

\section{Bio-inspired robotic modules}

\subsection{Bio-inspired mechanical cells}

Stem cells have the remarkable potential to be developed into different cell types during their life cycles, and under certain physiological or experimental conditions, they can be induced to become tissue- or organ-specific cells with special functions. Inspired by the evolution of stem cell, this paper proposes the concept of mechanical stem cells that are immature and have the potentiality to evolve into various robotic modules, such as bone, joint and muscle etc. A typical example of such a concept is illustrated in Fig. 1 where a mechanical stem cell is designed as a solid plate without any connections, in this sense, the cell is immature and its structure and function are indeterminate. However, with further evolution, the stem cell can grow connections at its edges and develop itself into mature mechanical cells. The connections can be of different types and two of them are considered in this paper, they are namely the male connection denoted as $\mathrm{M}$ and the female connection denoted as F. With the connections, mechanical cells such as FNMN cell and FFMM cell can be developed as shown in Fig. 1, where $\mathrm{N}$ standing for no connection. In Fig. 1, the FNMN cell consists of one mechanical stem cell and two connections, i.e. one female connection and one male connection; the FFMM cell has four connections which are arranged as F, F, M and M in clockwise sequence.

\subsection{Development of mechanical bones}

The FNMN and FFMM mechanical cells can further evolve themselves into mechanical bones forming structure modules that can be used as links or rigid bodies for constructing bioinspired robotic arms. For example, the FNMN and FFMM mechanical cells can interact and grow into different mechanical bones such as the cubic bone, cuboid bone and polyhedral bone that are indicated in Fig. 2.

The cubic bone is a stable structure consisting of six FFMM cells, and by adding four more FFMM cells to a cubic bone, a cuboid bone can be generated as shown in Fig. 2b, the length of the cuboid bone is doubled that of the cubic bone. In theory, more FFMM cells may be incorporated in order 


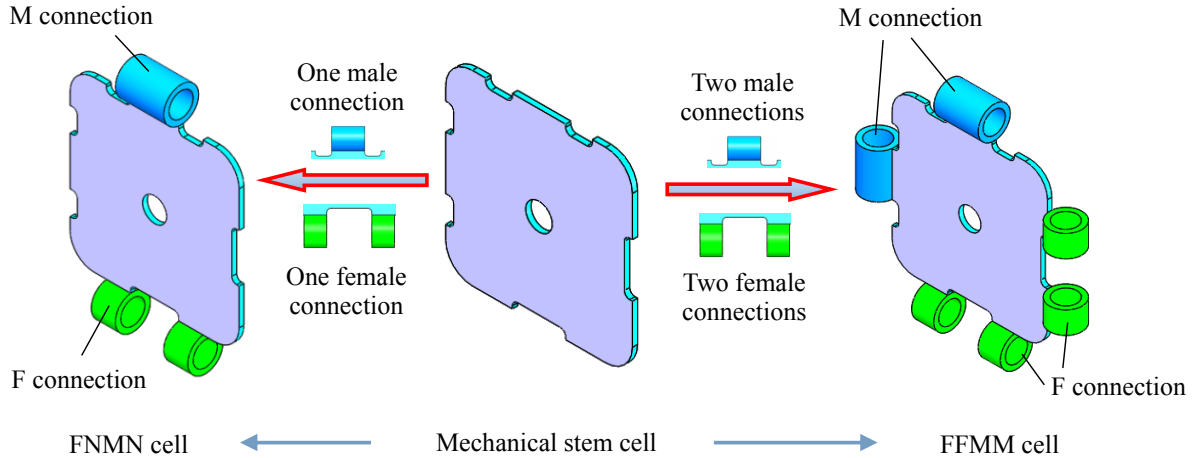

Figure 1. Bio-inspired mechanical cell.

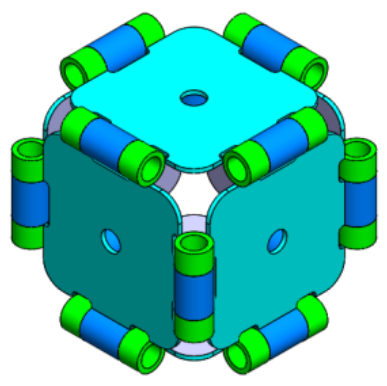

(a) Cubic bone

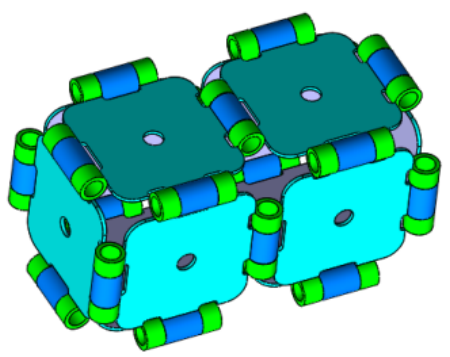

(b) Cuboid bone

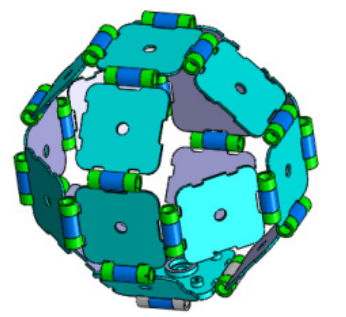

(c) Polyhedral bone

Figure 2. Bio-inspired mechanical bones.

to extend the cuboid bones. Figure $2 \mathrm{c}$ shows a polyhedral bone consists of eight FFMM cells and twelve FNMN cells. Compared to the cuboid bone, a polyhedral bone has more inner space which can be used to install and store actuators, sensors and other member modules.

A bio-inspired mechanical bone developed through the mechanical cells have three advantages. First, its structure is simple, modular and apt-to-be fabricated. Second, its structure is reconfigurable and flexible. Third, the inner hollow space of these mechanical bones can be used to accommo- date sub modules of robot, which is very useful for some special applications such as space technology.

\subsection{Bio-inspired Sarrus-linkage-based mechanical muscle}

Sarrus linkage (Sarrus, 1853) is a one degree of freedom spatial linkage that was invented by Pierre Frédéric Sarrus (1798-1861) in 1853, that can transfer rotary motion into linear motion, or inversely transfer the linear motion into standard rotary motion (Chen et al., 2013); sometimes it is also referred to as spatial crank mechanism. It provides a great variety of possible motions and may accomplish complex tasks beyond what planar linkages are capable of as indicated in He et al. (2014) and Li et al. (2013). The classic Sarrus linkage is a two-limb six-bar linkage of which each limb contains three parallel revolute joints, with the first joint of both limbs lying on the base plane and the third joint of both limbs lying on the platform plane.

Based on the Sarrus linkage, we can artificially nurture the FNMN and FFMM mechanical cells into a bio-inspired mechanical muscle that is composited of six mechanical cells connected by six revolute joints as indicated in Fig. 3a. Like the Sarrus linkage, the mechanical cells are jointed to form two sets of limbs, each of which being a four-link chain. The first chain is comprised of two FFMM cells as the bottom end labelled 1 and the top end labelled 4, and two FNMN cells serving as side links 2 and 3; the cells are connected by three parallel joints whose axes are denoted as A, B and C as indicated in Fig. 3a. Similarly, the other branch is composed of two FFMM cells serving as bottom end 1 and top end 4 which are of duplicates of the first branch, and two FNMN cells acting as side links 5 and 6 ; the links are joined by three parallel revolute joints whose axes are D, E and F, respectively.

To meet the requirement of practical engineering applications, a robuster Sarrus-linkage-based mechanical muscle is symmetrically developed by integrating four additional FNMN cells into the standard Sarrus structure associated with six extra joints, as illustrated in Fig. $3 b$. This design 


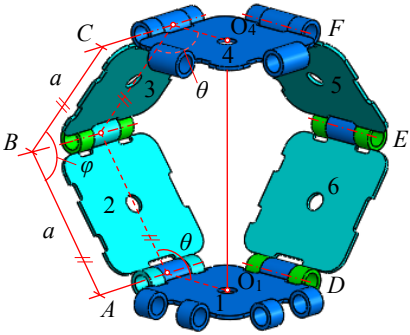

(a) Standard sarrus structure

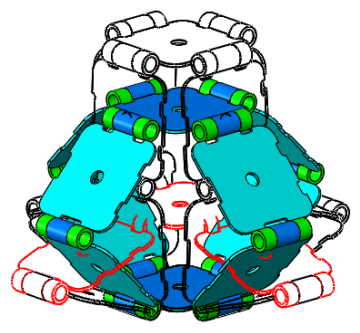

(b) Sarrus structure with redundant chains
Figure 3. Bio-inspired Sarrus-linkage-based mechanical muscle.

does not alter the motion performance and output characteristics of a standard Sarrus structure but provides a more stable and reliable structure which is capable of carrying more payload. As illustrated in Fig. 3b, assuming that the bottom cell is fixed, the top cell can only has translational motion with respect to the bottom cell. The lower-side cells rotates about the bottom cell, and the upper-side cells rotate respectively about their adjacent lower-side cells and translate with the top cell. Obviously, the linear motion of the two parallel ends leads to the circular motion of the joints; and on the other hand, the circular motion of the joints results in the linear motion between the two parallel ends. Hence, this mechanical muscle can not only be used as a translational joint, but also acts as a supporting structure to expand further the workspace of a robotic arm.

Further, given the structure parameters of the Sarrus structure as illustrated in Fig. 3a, there exist $\varphi+2 \theta=2 \pi$ and $d_{O_{1} O_{4}}=a \sqrt{2-2 \cos \varphi}$ with $d_{O_{1} O_{4}}$ standing for the distance between points $O_{1}$ and $O_{4}$.

\section{Design and kinematics of the bio-inspired robotic arm}

\subsection{Design of a bio-inspired modular 5-DOF robotic arm}

Based on the mechanical bones and muscle developed from the mechanical stem cells in the previous section, different type of robotic arms can be evolved and grown satisfying specific purpose and environment, and one bio-inspired modular-based 5-DOF robotic arm is developed in this paper as illustrated in Fig. 4. This bio-inspired robotic arm is composed of three bio-inspired bones, i.e. the shoulder bone, upper-arm bone and forearm bone, and two bio-inspired muscles as the upper-arm muscle and the forearm muscle, connected by three revolute joints, that is, the shoulder joint, the elbow joint and the wrist joint, and two rigid connections as the upper arm rigid connection and the forearm rigid connection. It can be seen that the robotic arm is purely developed from the mechanical stem cell presented in Sect. 2.1. Regarding the degrees of freedom of the robotic arm, there are three revolute joints, two 1-DOF Sarrus-linkage-based mus-

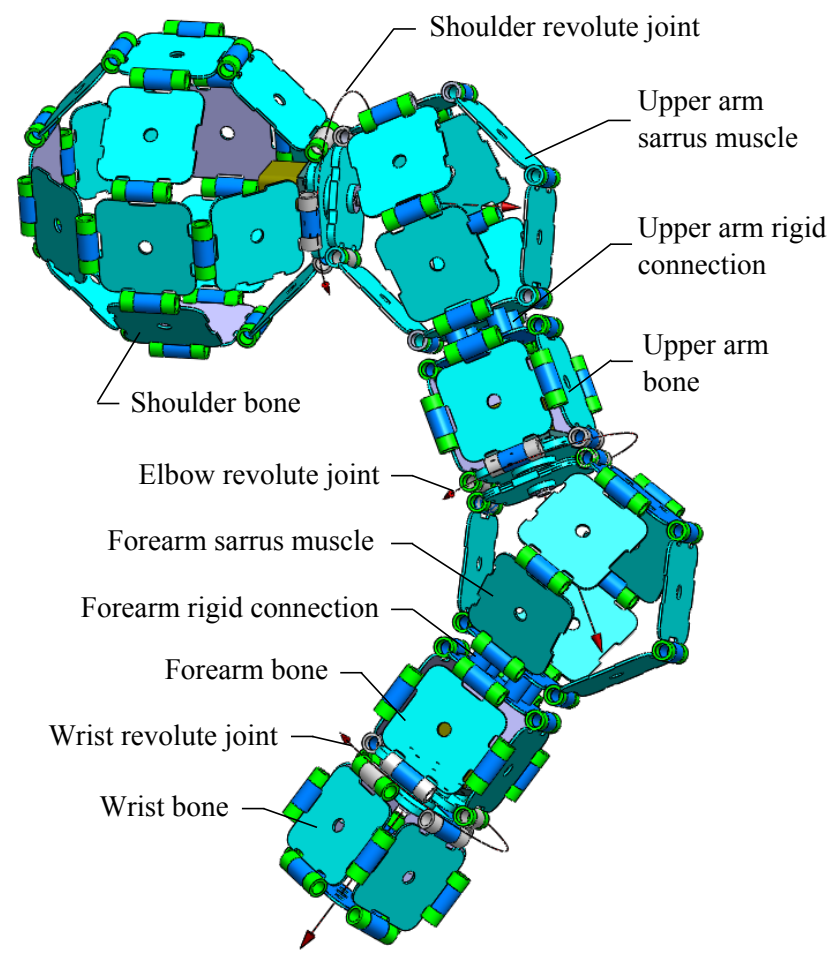

Figure 4. A bio-inspired modular 5-DOF robotic arm.

cles and two rigid connections, hence the total degrees-offreedom of the proposed robotic arm is five.

For this robotic arm, through the shoulder revolute joint, it can realize an circular $360^{\circ}$ rotation of upper arm; through the upper arm Sarrus-linkage-based muscle, it can realize a combined rotation and translation of the upper arm and below parts, and change the workspace of the arm; through the elbow revolute joint, it can realize an entire $360^{\circ}$ rotation of forearm and the parts below; through forearm Sarrus muscle, it can realize a combined rotational and translational motion of the forearm and parts below, and change its workspace; further, through the wrist revolute joint, it can realize circu$\operatorname{lar} 360^{\circ}$ rotation of the wrist bone.

As described above, the dexterous motion generated by the proposed robotic arm provides augmented workspace. Regarding its application, the shoulder can be fixed on the base or combined with the other robots, we can also directly connect the upper Sarrus arm to the base or the other robots with the shoulder revolute joint. By accurate controlling the motion of each joint, the robotic arm can execute specific mission satisfying various customer requirements.

\subsection{Forward kinematics}

As aforementioned, the bio-inspired 5-DOF robotic arm developed in this paper is evolved from the mechanical stem cell consisting of bone cells and Sarrus-linkage-based muscles. Kinematics of such a robotic arm is different from the 
conventional ones and is investigated in this section. In order to describe the position and orientation of the proposed robotic arm, a global coordinate system $\left\{O_{0}-x_{0} y_{0} z_{0}\right\}$ is located at the shoulder joint, and four body-attached coordinate systems are established at the upper arm muscle, upper arm born, forearm muscle and forearm born, respectively, as shown in Fig. 5. As the robotic arm includes both serial and parallel kinematic chains, the traditional D-H method cannot be directly used to analyse its kinematics. In this paper, a more general approach, that is, homogeneous coordinate transformation method is used. By firstly solving the transformation matrices between the coordinate systems, and consequently deriving the total transformation matrix, we can obtain a matrix representing the posture of the wrist bone with respect to the global coordinate system leading to the investigation of the workspace and inverse kinematics of the proposed robotic arm.

Considering the motion sequence of the robotic arm, it involves both translational and rotational transformation. Herein, the homogeneous transformation matrix for translation is denoted as $\operatorname{Trans}(a, b, c)$ with $a, b$ and $c$ being the coordinates of a displacement vector $a \boldsymbol{i}+b \boldsymbol{j}+c \boldsymbol{k}$, and the homogeneous transformation matrices for rotations about the $x, y$ and $z$ axes are denoted as $\operatorname{Rot}(x, \theta), \operatorname{Rot}(y, \theta)$ and $\operatorname{Rot}(z, \theta)$, respectively, as defined in Cai (2009). Further, for conciseness sake, the sine and cosine functions are denoted as $s$ and $c$, respectively.

As discussed above, the bio-inspired robotic arm is built by the FFMM and FNMN mechanical cells evolved from the mechanical stem cell, thus all the unit modules used to construct the robotic arm have the same structure parameters and we assume that lengths of the side and thickness of a unit module are respectively $a$ and $b$. According to the kinematics of Sarrus linkage, the angles $\theta_{i}$ and $\varphi_{i}(i=2$ and 4$)$ in the two Sarrus-linkage-based muscles in the robotic arm have the following relationship

$2 \theta_{i}+\varphi_{i}=2 \pi \quad(i=2$ and 4$)$.

Referring to Fig. 5, the position vector of the centroid point $\mathrm{M}$ in the wrist bone can be expressed in reference frame $\left\{O_{4}-x_{4} y_{4} z_{4}\right\}$ in homogeneous coordinate form as

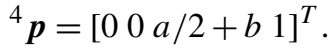

Coordinate system $\left\{O_{4}-x_{4} y_{4} z_{4}\right\}$ is transformed from frame $\left\{O_{3}-x_{3} y_{3} z_{3}\right\}$ by a rotation about the $y_{3}$ axis by an angle $\theta_{5}$ followed by another rotation about the $x_{4}$ axis by $-\pi / 2$, and subsequently a translation $\boldsymbol{q}_{34}$ along the $z_{4}$ axis. With respect to reference frame $\left\{O_{3}\right\}$, the translation $\boldsymbol{q}_{34}$ can be expressed as

$$
\begin{aligned}
& { }^{3} \boldsymbol{p}_{34}=\overline{O_{3} O_{4}}=\overline{O_{3} D}+\overline{D E}+\overline{E F}+\overline{F O_{4}} \\
& =\left[\begin{array}{llll}
a / 2 & 2 a \sin \theta_{4}+b & 0 & 1
\end{array}\right]^{T},
\end{aligned}
$$

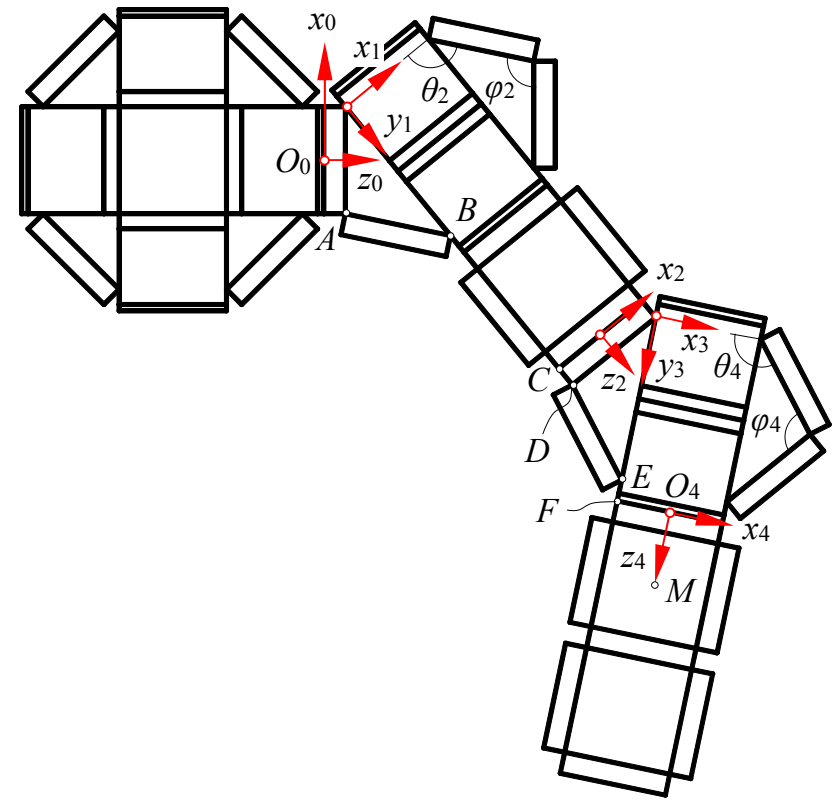

Figure 5. Geometry and coordinate systems of the robotic arm.

such that the homogeneous transformation matrix that defines reference frame $\left\{\mathrm{O}_{4}\right\}$ relative to reference frame $\left\{\mathrm{O}_{3}\right\}$ can be given as

$$
\begin{aligned}
\mathbf{T}_{34} & =\operatorname{Trans}\left({ }^{3} \boldsymbol{p}_{34}\right) \operatorname{Rot}\left(y_{3}, \theta_{5}\right) \operatorname{Rot}\left(x_{4},-\pi / 2\right) \\
& =\left[\begin{array}{cccc}
c \theta_{5} & -s \theta_{5} & 0 & a / 2 \\
0 & 0 & 1 & 2 a s \theta_{4}+b \\
-s \theta_{5} & -c \theta_{5} & 0 & 0 \\
0 & 0 & 0 & 1
\end{array}\right]
\end{aligned}
$$

Similarly, according to Fig. 5, posture of coordinate system $\left\{\mathrm{O}_{3}\right\}$ can be obtained from reference frame $\left\{\mathrm{O}_{2}\right\}$ by a rotation about the $z_{2}$ axis by angle $\theta_{3}$ with a following rotation about the $x_{2}$ axis by $\pi / 2$ and a translation given by vector $\boldsymbol{q}_{23}$. The translational vector $\boldsymbol{q}_{23}$ can be presented in reference frame $\left\{\mathrm{O}_{2}\right\}$ as

${ }^{2} \boldsymbol{p}_{23}=\left[\begin{array}{llll}a / 2 & b & 0 & 1\end{array}\right]^{T}$,

and therefore transformation matrix that defines frame $\left\{\mathrm{O}_{3}\right\}$ with respect to frame $\left\{\mathrm{O}_{2}\right\}$ can be obtained as

$$
\begin{aligned}
\mathbf{T}_{23} & =\operatorname{Trans}\left({ }^{2} \boldsymbol{p}_{23}\right) \operatorname{Rot}\left(z_{2}, \theta_{3}\right) \operatorname{Rot}\left(y_{2}, \theta_{4}-\pi\right) \operatorname{Rot}\left(x_{2}, \pi / 2\right) \\
& =\left[\begin{array}{cccc}
-c \theta_{3} c \theta_{4} & -c \theta_{3} s \theta_{4} & s \theta_{3} & a / 2 \\
-s \theta_{3} c \theta_{4} & -s \theta_{3} s \theta_{4} & -c \theta_{3} & 0 \\
s \theta_{4} & -c \theta_{4} & 0 & b \\
0 & 0 & 0 & 1
\end{array}\right]
\end{aligned}
$$


Further, by defining the displacement vector of the origin of frame $\mathrm{O}_{2}$ with respect to the origin of frame $\left\{O_{1}\right\}$ as

$$
\begin{aligned}
& { }^{1} \boldsymbol{p}_{12}=\overline{O_{1} O_{2}}=\overline{O_{1} A}+\overline{A B}+\overline{B C}+\overline{C O_{2}}
\end{aligned}
$$

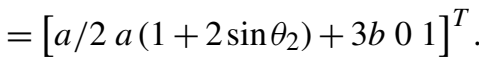

Transformation of coordinate system $\left\{O_{2}\right\}$ with respect to reference frame $\left\{O_{1}\right\}$ that is achieved by a rotation about the $x_{1}$ axis by $-\pi / 2$ and a translation with displacement vector ${ }^{1} \boldsymbol{p}_{12}$ can be expressed as

$$
\begin{aligned}
\mathbf{T}_{12} & =\operatorname{Trans}\left({ }^{1} \boldsymbol{p}_{12}\right) \operatorname{Rot}\left(x_{1},-\pi / 2\right) \\
& =\left[\begin{array}{cccc}
1 & 0 & 0 & a / 2 \\
0 & 0 & 1 & a\left(1+2 s \theta_{2}\right)+3 b \\
0 & -1 & 0 & 0 \\
0 & 0 & 0 & 1
\end{array}\right] .
\end{aligned}
$$

From Fig. 5, the displacement of the origin of reference frame $\left\{O_{1}\right\}$ with respect to the origin of frame $\left\{O_{0}\right\}$ can be given as

${ }^{0} \boldsymbol{p}_{01}=\left[\begin{array}{llll}a / 2 & b & 0 & 1\end{array}\right]^{T}$,

thus, the transformation from frame $\left\{O_{1}\right\}$ to frame $\left\{O_{0}\right\}$ which is performed by a rotation about the $z_{0}$ axis by angle $\theta_{1}$, a following rotation about the $x_{0}$ axis by $\pi / 2$, and a subsequent translation $\boldsymbol{p}_{01}$ can be derived as

$$
\begin{aligned}
\mathbf{T}_{01} & =\operatorname{Trans}\left({ }^{0} \boldsymbol{p}_{01}\right) \operatorname{Rot}\left(z_{0}, \theta_{1}\right) \operatorname{Rot}\left(y_{0}, \theta_{2}-\pi\right) \operatorname{Rot}\left(x_{0}, \pi / 2\right) \\
& =\left[\begin{array}{cccc}
-c \theta_{1} c \theta_{2} & -c \theta_{1} s \theta_{2} & s \theta_{1} & a / 2 \\
-s \theta_{1} c \theta_{2} & -s \theta_{1} s \theta_{2} & -c \theta_{1} & 0 \\
s \theta_{2} & -c \theta_{2} & 0 & b \\
0 & 0 & 0 & 1
\end{array}\right] .
\end{aligned}
$$

Finally, multiplying all the four transformation matrices in Eqs. (4), (6), (8) and (10), we can have the final coordinate transformation matrix relating frame $\left\{O_{4}\right\}$ to frame $\left\{O_{0}\right\}$ as

$\mathbf{T}_{04}=\mathbf{T}_{01} \mathbf{T}_{12} \mathbf{T}_{23} \mathbf{T}_{34}$

Furthermore, combining Eq. (2) and Eq. (11), the position vector of point $M$ relative to be base coordination system $\left\{O_{0}\right\}$ can be expressed as

${ }^{0} \boldsymbol{p}=\mathbf{T}_{04}{ }^{4} \boldsymbol{p}$.

According to Eqs. (2) and (12), it can be found that the wrist revolute joint has no effect on the position of the wrist bone centroid $M$, but it affects the orientation of wrist bone.

\subsection{Inverse kinematics}

Inverse kinematics provides background for position control of a robotic arm. Given the Cartesian coordinate of the endeffector in the transformation matrix $\mathbf{T}_{04}$, we attempt to derive the joint space formed by the five variables $\vartheta=\left\{\theta_{1}, \theta_{2}\right.$, $\left.\theta_{3}, \theta_{4}, \theta_{5}\right\}$. Since the proposed robotic arm is formed in a hybrid configuration, the inverse kinematics is highly nonlinear such that it is difficult to find closed-form solutions. Thus, in this section, a numerical approach is proposed for solving the inverse kinematics of this bio-inspired robotic arm.

Dividing matrix $\mathbf{T}_{04}$ into blocks, it has

$\mathbf{T}_{04}=\left[\begin{array}{cc}\mathbf{R}_{04} & \boldsymbol{q}_{04} \\ \mathbf{0} & 1\end{array}\right]$,

where, $\mathbf{R}_{04}=\left[\begin{array}{lll}\boldsymbol{x}_{04} & \boldsymbol{y}_{04} & z_{04}\end{array}\right]^{T}$ is the rotation matrix lying in $S O$ (3) defining orientation of the wrist bone with respect to the base frame, and $\boldsymbol{q}_{04}=\left[\begin{array}{lll}q_{x} & q_{y} & q_{z}\end{array}\right]^{T}$ is a vector in $\mathbb{R}^{3}$ that locates the position of the centroid $M$ of the wrist bone relative to the base frame.

Assuming that elements of the matrix in Eq. (13) are specified, equalizing Eqs. (11) and (13) leads to a set of non-linear equations containing 5 variables and 12 equations which are not all independent. In this way, the inverse kinematic problem of the proposed robotic arm is converted into finding solutions for the 12 dependent equations.

However, as aforementioned, due to the hybrid structure of the proposed bio-inspired robotic arm, there is no analytical solution for the set of non-linear transcendental equations, there exist only numerical solutions or approximate solutions. Further, unless in the case of special circumstances, there is no general direct method to get the numerical solution structure of non-linear equations, including the existence of solution and multiple solutions. At present, there are two approaches for obtaining the numerical solution, i.e. the indirect method based on fixed point theorem, and the optimization method based on variational principle, both of these methods are based on iterative numerical solution adopting the strategy of the "time for accuracy" termed in Zhou et al. (2008). Considering that the kinematics equations to be solved herein is a hyper-redundant non-linear transcendental one without direct solution available, we convert this problem into a global optimization problem based on optimization methods as follows.

Given elements to matrix $\mathbf{T}_{04}$ in Eq. (13) and equalizing it with Eq. (11), a set of dependent equation can be formed in terms of the five variables as

$$
\left\{\begin{array}{c}
f_{1}\left(\theta_{1}, \theta_{2}, \theta_{3}, \theta_{4}, \theta_{5}\right)=0 \\
f_{2}\left(\theta_{1}, \theta_{2}, \theta_{3}, \theta_{4}, \theta_{5}\right)=0 \\
\cdots \\
f_{12}\left(\theta_{1}, \theta_{2}, \theta_{3}, \theta_{4}, \theta_{5}\right)=0
\end{array},\right.
$$

where the joint space $\vartheta=\left\{\theta_{1}, \theta_{2}, \theta_{3}, \theta_{4}, \theta_{5}\right\}$ contains the five variables whose variation ranges are defined as

$\pi / 2 \leq \theta_{2}, \theta_{4} \leq \pi$ and $-\pi \leq \theta_{1}, \theta_{2}, \theta_{3} \leq \pi$. 
In order to convert the equation solving problem into an equivalent optimization problem, Eq. (14) is converted to construct an energy function as

$P(\vartheta)=\sum_{i=1}^{12}\left|f_{i}\left(\theta_{1}, \theta_{2}, \theta_{3}, \theta_{4}, \theta_{5}\right)\right|$.

Thus, the problem of solving non-linear transcendental equations is transformed into the problem of solving the energy function complying with a given constraint. That is, given a sufficiently small positive number $\varepsilon$, the aim is to search for a set of values $\vartheta^{*}\left(\theta_{1}, \theta_{2}, \theta_{3}, \theta_{4}, \theta_{5}\right)$ such that $P\left(\vartheta^{*}\right)<\varepsilon$ holds, and as a result, $\vartheta^{*}\left(\theta_{1}, \theta_{2}, \theta_{3}, \theta_{4}, \theta_{5}\right)$ provides as a set of approximate solutions for the above nonlinear transcendental equations.

The numerical solutions of non-linear transcendental equations can be implemented in three steps as presented in Lu et al. (2008):

1. the existence of solutions: checking whether the equations have solutions;

2. solution isolation: dividing the solution interval into smaller sub-intervals, for each sub-interval, there may exist a solution or not, and if so, we can refer to any point in the sub-intervals as an approximation solution;

3. solution precise: efforts to improve the accuracy of the approximation solution, make it satisfy a certain accuracy requirement.

Considering the approach of solving non-linear equations based on global optimization method, a numerical iterative algorithm is proposed with its procedures shown in Fig. 6 and the specific process is interpreted as follows.

Firstly, define the maximum number of iteration $N$ and the threshold error $\varepsilon$ : if the calculation accuracy reaches the threshold error $\varepsilon$, stop the operation and the corresponding set of variable obtained may provide a set of inverse kinematic solutions; if the number of operations has reached the maximum number of iteration $N$, even if accuracy error is larger than the threshold, also stop operations. Obviously, if the number of operations has reached the maximum number of iteration, and the calculation error is still big, this means that the accuracy of the solutions is not high enough to meet the threshold, we need to increase the maximum number of iteration $N$ so as to achieve a set of more precise solutions.

Secondly, allocate values of the five variables $\theta_{1}, \theta_{2}, \theta_{3}$, $\theta_{4}$ and $\theta_{5}$ according to their individual intervals defined in Eq. (15), and substitute them into the energy function $P(\vartheta)$, then perform the iterative operation and select the minimum value of the energy function $P(\vartheta)$ searching for the optimized set of variable values $\left(\theta_{k 1}, \theta_{k 2}, \theta_{k 3}, \theta_{k 4}, \theta_{k 5}\right)$ that leads to minimum value of the energy function denoted as $P(\vartheta)_{\min }$.

Thirdly, continue allocating the five variables $\theta_{1}, \theta_{2}, \theta_{3}, \theta_{4}$ and $\theta_{5}$ in a smaller interval near $\left(\theta_{k 1}, \theta_{k 2}, \theta_{k 3}, \theta_{k 4}, \theta_{k 5}\right)$, and

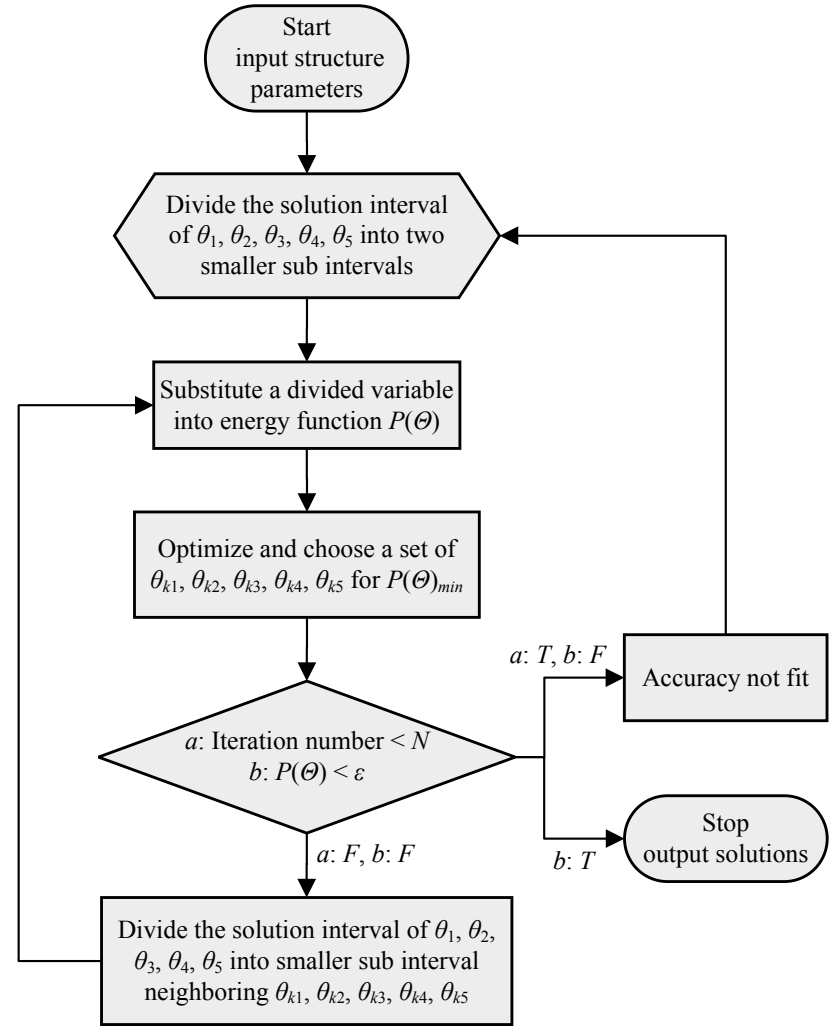

Figure 6. Flow chart for numerical solution of the proposed inverse kinematics.

running iterative operations. This will result in better minimum energy function value $P\left(\vartheta^{*}\right)$ and the refined solutions for the five variables.

Repeat the above steps until the value of energy function is less than the threshold error or the iteration times reached the maximum number, the final output of the five variables $\theta_{1}, \theta_{2}$, $\theta_{3}, \theta_{4}$ and $\theta_{5}$ will be a set of optimal solutions for the nonlinear equations, that is, the inverse kinematic solution of the proposed robotic arm.

Obviously, this algorithm can reliably obtain a set of solutions satisfying the equations through a series of continuous divide and iteration. To seek a more accurate solution, more divided intervals and iteration times are required, which naturally increases the operation time. It can be found that the above algorithm can satisfy the general application requirements without involving any kind of complex algorithm such as genetic algorithm or neural networks. In this paper, the results obtained indicate that this algorithm is sufficient to compute the inverse kinematics and provide results for position and orientation control, the results are indicated by numerical simulation and physical prototype validation. 
(a) 3D workspace

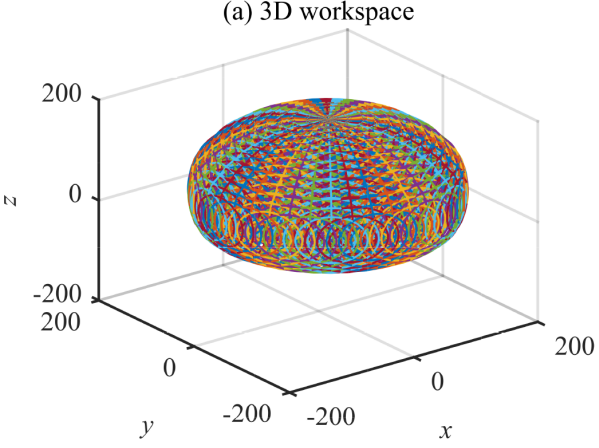

(c) Workspace in $x-z$ plane

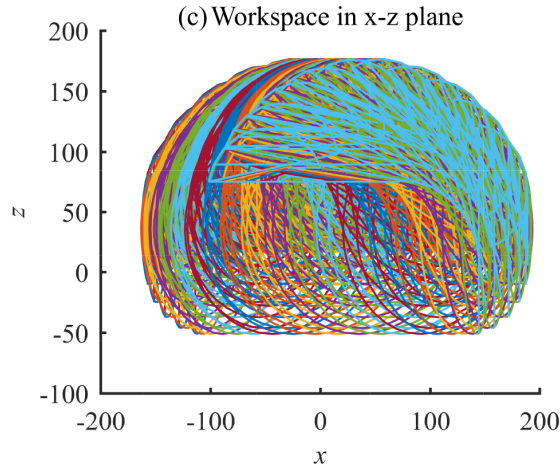

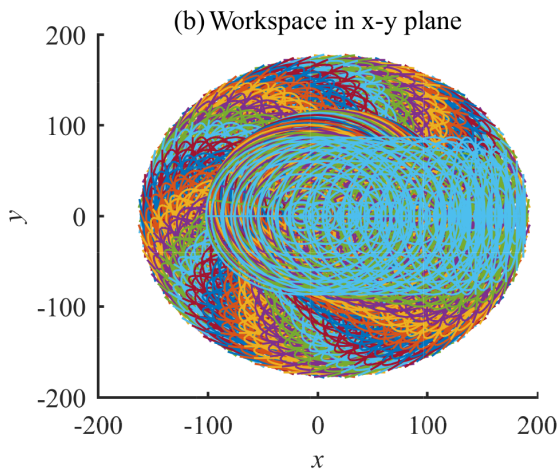

(d) Wrokspace in y-z plane

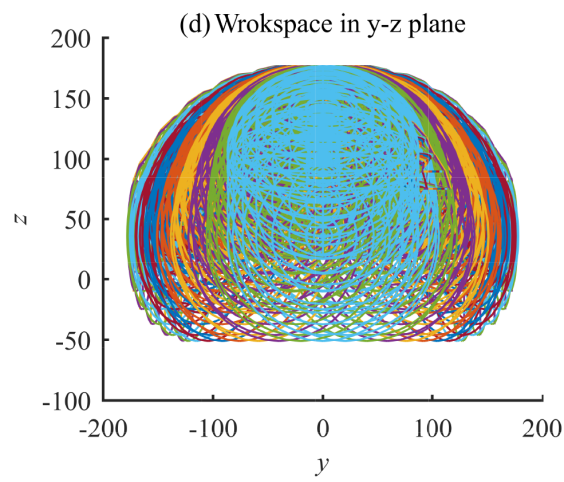

Figure 7. Workspace of the bio-inspired robotic arm.

\section{Numerical simulation and prototype of the robotic arm}

\subsection{Workspace and the typical configurations}

Based on the transformation matrix of the wrist bone centroid $M$ given in Eq. (12), using Matlab package, we can plot the trajectory of point $M$ relative to the fixed reference frame $\left\{O_{0}\right\}$, that is, workspace of the wrist bone centroid $M$ relative to the universal coordinate system, as shown in Fig. 7. In this example, the size of each stem mechanical cell is $a \times a \times b=30 \mathrm{~mm} \times 30 \mathrm{~mm} \times 6 \mathrm{~mm}$, in addition, the geometric collisions among member modules during spatial motion are not considered.

Figure $7 \mathrm{a}$ and $\mathrm{b}$ show that the projection of the workspace of the robotic arm in any plane that is perpendicular to the $z$ axis is symmetric, the arm can make a whole circular motion around the $z$ axis without any interference. Figure $7 \mathrm{~b}$ and $\mathrm{c}$ show that the wrist can perform both forward and backward motions in a certain range in the negative direction of the $z$ axis, which provides potential capability of mimicking the motion of a human arm.

The workspace of the robotic arm in Fig. 7 also demonstrates that, without considering the size of the shoulder, when all the Sarrus-linkage-base muscles are fully retracted, the entire arm length in the $z$ axis direction is $108 \mathrm{~mm}$, while the wrist centroid $M$ can reach in a the range from -71 to $200 \mathrm{~mm}$ along $z$ axis, as illustrated in Fig. 7c and d. Like- wise, when retracted, the position of wrist centroid $M$ in the $x$ - and $y$-direction can be zero, and the actual workspace is in the range from -175 to $175 \mathrm{~mm}$ along the $x$ axis and the $y$ axis, respectively.

In addition, Fig. 8 demonstrates the typical configurations and workspace augmentation principle of the proposed robotic arm. Figure 8a shows one extreme phase of the robotic arm with two Sarrus-linkage-based muscles being fully retracted, compared with the fully expanded phase indicated in Fig. 8b and the intermediate phase in Fig. 8c, the robotic arm has the smallest volume of workspace at this phase, and in this configuration, the lengths of three equivalent links $l_{1}, l_{2}$ and $l_{3}$ are $l_{1}=\sqrt{a^{2}+(a+4 b)^{2}}$, $l_{2}=\sqrt{a^{2}+4 b^{2}}$ and $l_{3}=a / 2+b$, respectively; where, $a$ and $b$ are respectively the side length and thickness of the mechanical stem cells. Figure $8 \mathrm{~b}$ shows another extreme phase of the robotic arm with the two Sarrus-linkage-based muscles being fully expanded, compared with Fig. 8(a), it can be found that the lengths between $O_{0}$ and $O_{2}$, and between $O_{2}$ and $O_{4}$ increase, such that the lengths of the three equivalent links become $l_{1}=\sqrt{(a / 2+b)^{2}+(5 a / 2+3 b)^{2}}$, $l_{2}=\sqrt{(a / 2+b)^{2}+(3 a / 2+b)^{2}}$ and $l_{3}=a / 2+b$. Figure $8 \mathrm{c}$ shows an intermediate phase in which configuration the lengths of the three equivalent links are $l_{1}=$ $\sqrt{(a / 2(1-c \alpha)+b s \alpha)^{2}+(a(1+3 s \alpha / 2)+b(3-c \alpha))^{2}}$, $l_{2}=\sqrt{(a / 2(1-c \eta)+b s \eta)^{2}+(3 a / 2 s \eta-b c \eta)^{2}}$ and $l_{3}=$ $a / 2+b$. 


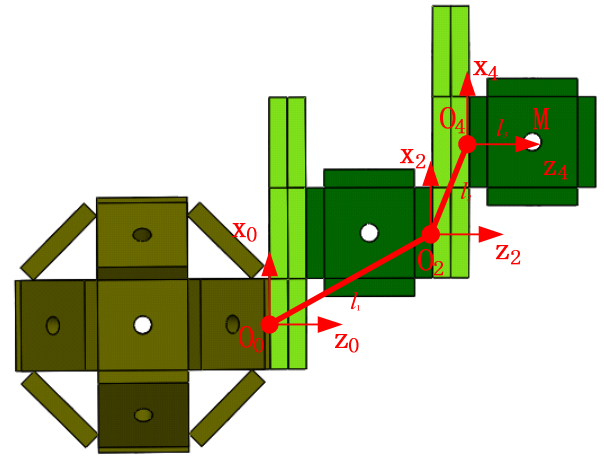

(a) Fully retracted configuration

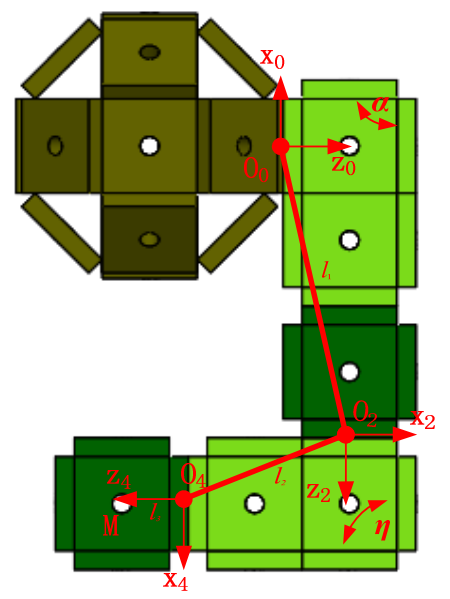

(b) Fully expanded configuration

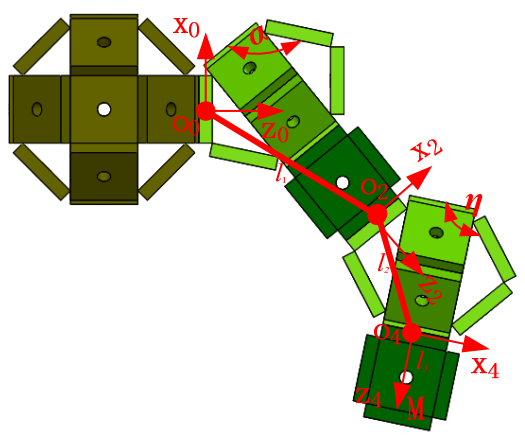

(c) Intermediate phase

Figure 8. Geometric configurations of the bio-inspired robotic arm.

Based on the changes of the lengths of the three equivalent links $l_{1}, l_{2}$ and $l_{3}$, the workspace augmentation function of the two Sarrus-linkage-based muscles can be formulated and illustrated in Fig. 9.

Figure 9a shows the relationship between length of the equivalent link $l_{1}$ and the configuration angle $\alpha$ of the upper arm Sarrus-linkage-based muscle. It can be found that as the upper arm muscle transform from the fully retracted

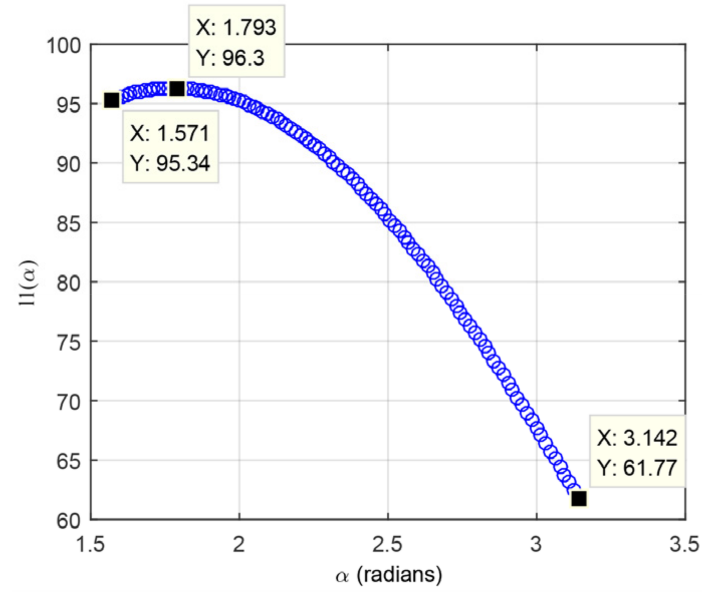

(a) Length change of upper arm muscle

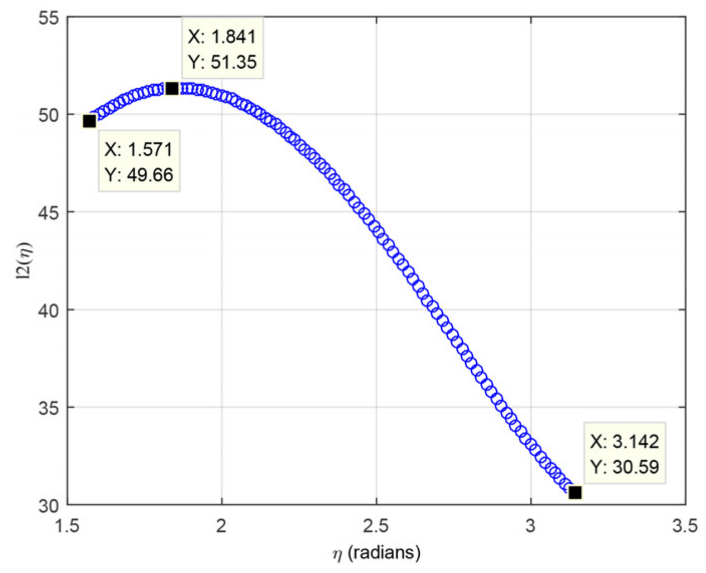

(b) Length change of forarm muscle

Figure 9. Workspace augmentation function of the two Sarruslinkage-based muscles.

phase to the fully expanded phase, the actual length of link $l_{1}$ increases from 61.77 to $95.34 \mathrm{~mm}$ with the maximum length of $96.33 \mathrm{~mm}$; which provides an extra $34 \mathrm{~mm}$ flexible length to the upper arm to achieve a linear motion along the $z$ axis.

Similarly, as shown in Fig. 9b, as the forearm Sarruslinkage-based muscle transforms from the fully retracted phase to the fully expanded phase, the actual length of the equivalent link $l_{2}$ increase from 30.59 to $49.66 \mathrm{~mm}$, and the maximum length is $51.35 \mathrm{~mm}$, which implies that an extra $20 \mathrm{~mm}$ flexible length is provided to the forearm for the linear motion along the $z_{4}$ axis.

Hence, with the two Sarrus-linkage-based muscles integrated in this novel bio-inspired robotic arm, it can break through the limitation of conventional robotic structure and enlarge its workspace, which makes the robotic arm particularly suitable for performing large-scale motion in a compact space, not only as a general industrial robotic arm, but also 
as a device carried by mobile robot to perform investigation, detection, rescue, transportation and other tasks.

\subsection{Numerical solution for the inverse kinematics}

Further, in order to validate the feasibility and accuracy of the proposed algorithm for inverse kinematic analysis, one numerical example is provided in this section. The validation method and process is: first, give an initial value for the five variables $\theta_{1}, \theta_{2}, \theta_{3}, \theta_{4}$ and $\theta_{5}$, the two structure parameters $a$ and $b$ of the mechanical stem cell, and substitute them into the forward kinematics matrix in Eq. (11) to achieve the transformation matrix; second, by equating the specified transformation matrix to Eq. (13), construct a set of non-linear transcendental equations containing 12 equations; third, by using the algorithm presented in Sect. 3.3, solve the equations and check the group of optimal solutions and minimum energy function value, this leads to the appropriate inverse kinematic solutions of the robotic arm.

Here, we assume that the mechanical stem cell has structure parameters $a=30 \mathrm{~mm}, b=6 \mathrm{~mm}$, and a set of values within the ranges of the five joint variables is given as

$\vartheta=\left(\theta_{1}, \theta_{2}, \theta_{3}, \theta_{4}, \theta_{5}\right)=(\pi / 1.5, \pi / 1.2,-\pi / 2,-\pi / 6, \pi / 3)$.

Firstly, substituting the variables in Eq. (17) into the transformation matrix in Eq. (11), the transformation matrix for representing the wrist bone coordinate system with respect to the global coordination system can be specified as

$\mathbf{T}_{04}=\left[\begin{array}{cccc}0.6250 & 0.3460 & -0.6998 & -43.3841 \\ -0.6495 & -0.2667 & -0.7120 & -96.1147 \\ -0.4330 & 0.8995 & 0.0580 & 100.5428 \\ 0 & 0 & 0 & 1\end{array}\right]$

Then, by equating matrices Eqs. (18) and (13), a set of non-linear transcendental equation containing 5 variables and 12 equations can be constructed.

Subsequently, substituting the above matrix into the algorithm developed in Matlab ${ }^{\text {TM }}$ program, and setting subdivision number as $n=48$, the threshold error $\varepsilon=0.5$ and the maximum number of iterations $N=20$, we get a set of inverse kinematic solutions as

$$
\begin{aligned}
\vartheta & =\left(\theta_{k 1}, \theta_{k 2}, \theta_{k 3}, \theta_{k 4}, \theta_{k 5}\right) \\
& =(2.0982,2.6129,-1.6797,-0.3984,1.0433)
\end{aligned}
$$

where, the number of iterations is 16 , the minimum energy function value $P(\vartheta)_{\min }=0.2250$, such that $P(\vartheta)_{\min }<\varepsilon$ and therefore the operation terminates.

Finally, by substituting the results in Eq. (19) into the transformation matrix in Eq. (11), we can get a optimized numerical transformation matrix as

$\mathbf{T}_{04}^{\prime}=\left[\begin{array}{cccc}0.6645 & 0.3459 & -0.6624 & -43.3883 \\ -0.6073 & -0.2667 & -0.7484 & -96.1120 \\ -0.4356 & 0.8996 & 0.0328 & 100.5100 \\ 0 & 0 & 0 & 1\end{array}\right]$
Table 1. Errors of the inverse kinematic solutions.

\begin{tabular}{lllll}
\hline Element & $t_{11}$ & $t_{12}$ & $t_{13}$ & $t_{14}$ \\
Error & $6.32 \%$ & $0.00 \%$ & $5.34 \%$ & $0.00 \%$ \\
Element & $t_{21}$ & $t_{22}$ & $t_{23}$ & $t_{24}$ \\
Error & $6.50 \%$ & $0.00 \%$ & $5.11 \%$ & $0.00 \%$ \\
Element & $t_{31}$ & $t_{32}$ & $t_{33}$ & $t_{34}$ \\
Error & $0.60 \%$ & $0.00 \%$ & $43.45 \%$ & $0.00 \%$ \\
\hline
\end{tabular}

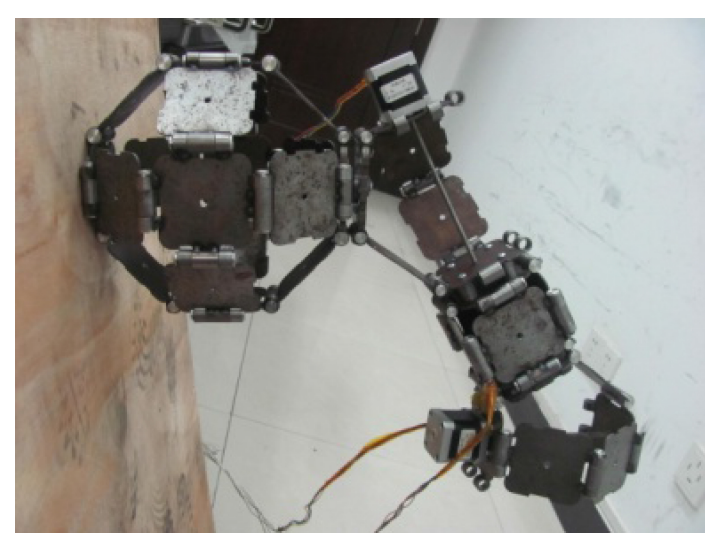

Figure 10. Prototype of the proposed bio-inspired robotic arm.

Comparing the results in Eq. (20) with the specified values in Eq. (18) as show in Table 1, it can be found that except for the element $t_{33}$, the errors are within the tolerance ranges, and the minimum value of energy function $P(\vartheta)$ is within the acceptable range. Therefore, Eq. (20) can be accepted as a set of inverse kinematic solutions for the robotic arm. At the same time, the results show that the numerical iteration method developed for the non-linear transcendental equations of robotic arm is valid and feasible.

\subsection{Prototype and validation}

Based on the forward and inverse kinematic analysis of the bio-inspired robotic arm, the mechanical cells were designed and fabricated, and a prototype of the proposed 5DOF robotic arm was constructed, assembled and integrated with low-lever control system as illustrated in Fig. 10. It can be seen that, the mechanical system was completely built by using only the FFMM and FNMN cells that are developed from the mechanical stem cell, the three revolute joints at the shoulder, elbow and wrist are driven by rotary stepper motors, and the Sarrus-linkage-based muscles are driven by linear motors.

To validate the forward and inverse kinematic models of the robotic arm, a virtual obstacles is designed to construct obstacle avoidance motion test of the robotic arm. As shown in Fig. 11a, the robotic arm is in the rear of a cuboid obstacle, the purpose of obstacle avoidance motion test is to locate the robotic arm in front of the obstacle without any interference. 

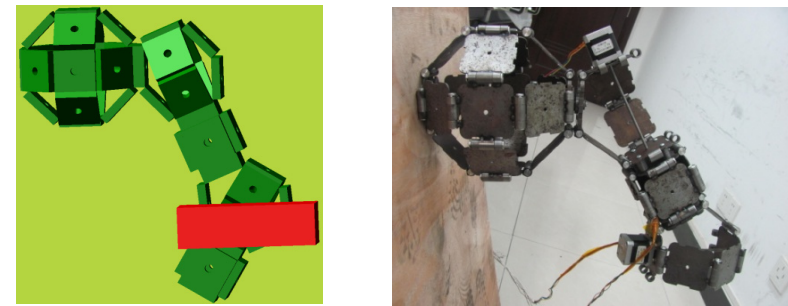

(a) Initial configuration of the robot
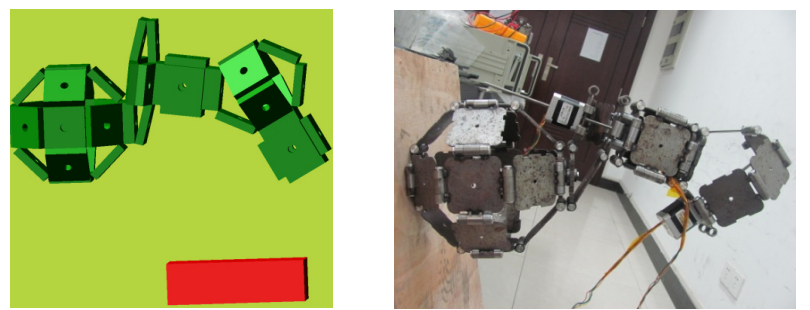

(b) Robotic arm shrinking
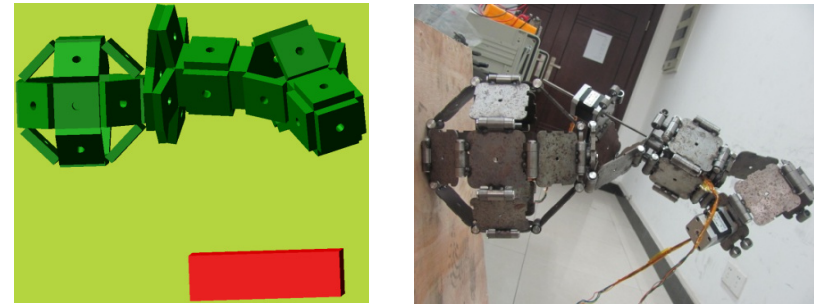

(c) Robotic arm swinging
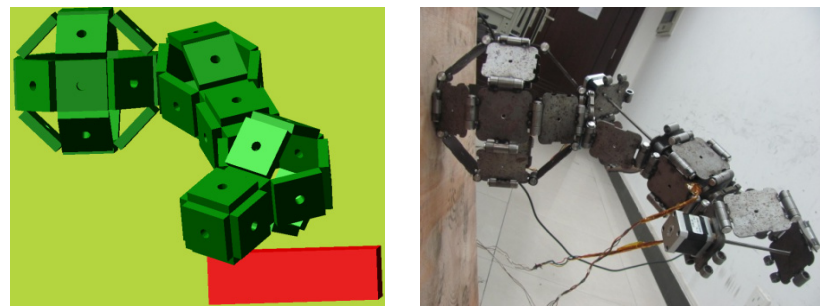

(d) Robotic arm expanding

Figure 11. Obstacle avoidance motion test of the robotic arm.

It can be seen that if the shoulder joint rotates directly, the whole arm will swing toward the obstacle, which may lead to an interference between the arm and the obstacle. Therefore, in order to avoid potential collision with the obstacle, a simple but efficient motion control strategy is used based on the property of the Sarrus-linkage-based muscles. Figure 11a shows the initial configuration of the robot in simulation corresponding with the physical test. By only driving the upper arm Sarrus-linkage-based muscle, the upper arm shrinks and the arm arrives at a position over the obstacle, as shown in Fig. 11b. Following a rotation of the shoulder's revolute joint, the robotic arm swings ahead and points in the front direction of the obstacle, as shown in Fig. 11c. Finally, by releasing the upper arm muscle, the upper arm expands to make the robotic arm reach the desired position avoiding the obstacle, as illustrated in Fig. 11d.
Initial tests indicate that the proposed robotic arm can not only perform the functions desired in the design but also overcome obstacles through the shrinking motion of the upper arm and forearm Sarrus-linkage-based muscles, which greatly simplifies control strategy and reduces the financial cost for establishing complex control system.

\section{Conclusions}

In this paper, a novel bio-inspired robotic arm was for the first time proposed and presented. This robotic arm was designed and developed based on a single type of mechanical stem cells.

Inspired by the function and characteristics of the stem cell but different from the other robot "cell" or "molecule", the mechanical stem cell presented in this paper is simple but capable of evolving into different functional cells, bones and muscles. Using the bones and a mechanical muscle developed based on the Sarruse linkage, a 5-DOF bio-inspired robotic arm was designed and its associated kinematics was investigated. In order to solve the inverse kinematics of the proposed robotic arm, an optimization-method-based numerical iterative algorithm was proposed and verified with a numerical example and computer simulations. Further, a physical prototype of the proposed 5-DOF robotic arm was developed and initial tests were carried out to validate the correctness of forward kinematics and the applicability of inverse kinematics solving algorithm.

Overall, the paper has indicated that the stem-cell inspired pure mechanical stem cell has parallels in biology and provides a flexible modular way to build mechanical bones and muscles for robotic arm development. Advantages of the proposed bio-inspired robotic arm can be summarized in three aspects: first, its structure is simple, modular and apt to be fabricated; second, its structure is reconfigurable and flexible; and third, the inner hollow space of these robot bones can be used to settle sub modules of robot, which is very useful for some special application such as space technology.

Author contributions. Designed and developed the robotic arm: Zirong Luo. Kinematics Analysis: Zirong Luo, Jianzhong Shang, Guowu Wei and Lei Ren. Simulation ad prototype: Zirong Luo, Jianzhong Shang. Wrote the paper: Zirong Luo, Jianzhong Shang, Guowu Wei and Lei Ren.

Acknowledgements. The author wishes to thank Ernest Appleton, Bo Liao, Yunkai Yang and Jun Zhang for their valuable contributions in developing the prototype.

Edited by: K. Mianowski

Reviewed by: L. Bruzzone and one anonymous referee 


\section{References}

Acaccia, G., Bruzzone, L., and Razzoli, R.: A modular robotic system for industrial applications, Assembly Automation, 28, 151$162,2008$.

Cai, Z.: Robotics, 2nd Edn., Tsinghua University Press, Beijing, 2009.

Chen, G., Zhang, S., and Li, G.: Multi-stable behaviors of compliant Sarrus mechanisms, J. Mech. Robot., 5, 021005, doi:10.1115/1.4023557, 2013.

Fukuda, T. and Kawauchi, Y.: Robotic system (CEBOT) as one of the realization of self-organizing intelligent universal manipulator, in: Proceedings IEEE Conference on Robotics and Automation, Cincinnati, Ohio, 662-667, 1990.

Fukufda, T. and Nakagawa, S.: Dynamically reconfigurable robotic system, in: Proceedings IEEE Conference on Robotics Automation, Franklin Plaza Hotel, Philadelphia, Pennsylvania, 15811586, 1988.

Gavin, D. and Luis, R.: Biologically inspired telescopingactive suspension arm vehicle: Preliminary results, in: IEEE/ASME International Conference on Advanced Intelligent Mechatronics, Montreal, Canada, 1380-1384, 2010.

Guan, Y., Jiang, L., Zhang, K. X., Qiu, J., and Zhou, X.: 1-DOF joint modules and their applications in new robotic systems, in: IEEE International Conference on Robotics and Biomimetics, Bangkok, 1905-1910, 2009.

Guckert, M. and Naish, M. D.: Design of a novel 3 degree of freedom robotic joint, in: IEEE/RSJ International Conference on Intelligent Robots and Systems, NJ, USA, 5146-5152, 2009.

He, X., Kong, X., Chablat, D., Caro, S., and Hao, G.: Kinematic analysis of a single-loop reconfigurable $7 \mathrm{R}$ mechanism with multiple operation modes, Robotica, 32, 1171-1188, 2014.

Jin, Z. and Gao, F.: Novel 6-SPS parallel3-dimensional platform manipulator and its force/motion transmission analysis, Chinese J. Mech. Eng., 15, 298-302, 2002.

Katie, L. and Robert, J.: Myriapod-like ambulation of a segmented micro robot, Autonomous Robots, 31, 103-114, 2011.

Kotay, K. and Rus, D.: Locomotion versatility through selfreconfiguration, Robot. Autonom. Syst., 2, 17-23, 1999.

Lee, C.-C.: Kinematic analysis and dimensional synthesis of general-type Sarrus mechanism, JSME Int. J. Ser. C, 39, 790$799,1996$.
Li, J., Zhang, G., Muller, A., and Wang, S.: A family of remote centerof motion mechanisms basedon intersecting motion planes, T. ASME J. Mech. Design, 135, 091009, doi:10.1115/1.4024848, 2013.

Liu, H., Huang, T., Mei, J., Zhao, X., Chetwynd, D. G., Li, M., and Hu, S.: Kinematic design of 5-DOF hybrid robot with largeworkspace/limb-stroke ratio, T. ASME J. Mech. Design, 129, 530-537, 2006.

Lu, T., Kang, Z., and Fang, X.: Numerical calculation method, Tsinghua University Press, Beijing, 2008.

Ranjana, S., Srinath, A., and Richard, G.: Towards a 3g crawling robot through the integration of micro robot technologies, in: IEEE International Conference on Robotics and Automation, Orlando, Florida, USA, 296-302, 2006.

Rus, D. and Vona, M.: Self-reconfiguration planning with compressible unit module, in: IEEE Proceeding of International Conference on Robotics and Automation, Detroit, MI, USA, 25132520, 1999.

Sarrus: Note sur la transformation des mouvements rectilignes alternatifsen mouvements circulaireset re ciproquement, Acad. Sci C. R. Hebd. Seances Acad. Sci., 36, 1036-1038, 1853.

Shammas, E., Wolf, A., and Choset, H.: Three degrees-offreedomjoint for spatial hyper-redundant robots, Mech. Mach. Theory, 41, 170-190, 2006.

Siciliano, B. and Khatib, O. (Eds.): Springer handbook of robotics, Springer-Verlag, Berlin, Heidelberg, 2008.

Wurst, K. H. and Peting, U.: PKM concept for reconfigurable machine tools, in: The 3rd Chemnitz Parallel Kinematics Seminar, Zwickau, Germany, 63-66, 2002.

$\mathrm{Xi}, \mathrm{F} ., \mathrm{Xu}, \mathrm{Y}$. , and Xiong, G.: Design and analysis of a reconfigurable parallel robot, Mech. Mach. Theory, 41, 2006.

Yim, M., Duff, D., and Roufas, K.: PolyBot: a modular reconfigurable robot, in: Proceedings of the 2000 IEEE International Conference on Robots \& Automation, San Francisco, CA, USA, 514-520, 2000.

Yoshida, K., Hata, N., Oh, S., and Hori, Y.: Extended manipulability measure and application for robot arm equipped with bi-articular driving mechanism, in: Proc. 35th Annu. Conf. IEEE Ind. Electron. Soc., Porto, 3083-3088, 2009.

Zhou, G., Song, B., and Xie, J.: Numerical analysis, Higher Education Press, Beijing, 2008. 